Conclusion Our results show striking variation in multimorbid conditions by gender, deprivation and ethnicity, and highlight the importance of multimorbidity, in particular comorbid depression, as a leading cause of disability in the population. The findings highlight the importance of multi-morbidity, particularly comorbid depression, as the leading cause of disability among working age adults.

\section{OP29 DYNAMICS OF MULTIMORBIDITY IN ENGLAND BETWEEN 2004 AND 2019: A DESCRIPTIVE EPIDEMIOLOGY STUDY USING THE CLINICAL PRACTICE RESEARCH DATALINK}

A Head*, K Fleming, C Kypridemos, P Schofield, M O'Flaherty. Public Health and Policy, University of Liverpool, Liverpool, UK

10.1136/jech-2020-SSMabstracts.29

Background An estimated 25\% of GP patients within the UK have multimorbidity, a large proportion of which is attributable to non-communicable diseases, many of them preventable. The heterogeneity of existing study methodologies and definitions of multimorbidity limits comparisons to assess temporal trends. This study aims to use a large population-representative single dataset and disease list to describe changes over time in multimorbidity incidence and prevalence.

Methods We selected a random sample of $1 \mathrm{~m}$ adults from the Clinical Practice Research Datalink (CPRD Aurum database) registered at participating GP practices within England between 2004 and 2019. We used two measures of multimorbidity: a) basic multimorbidity: two or more chronic conditions; b) complex multimorbidity: at least three chronic conditions affecting at least three body systems. A multidisciplinary team discussed the list of chronic conditions of interest, including long-term mental health conditions and chronic infections.

Using standard formulae, we calculated crude and age-sex standardised annual multimorbidity prevalence and incidence to assess trends over time. We also calculated the average age of onset for basic and complex multimorbidity. Analyses were conducted using $\mathrm{R}$ v3.6.3.

Participants will be linked to quintiles of the 2015 Index of Multiple Deprivation as a measure of area-level socioeconomic deprivation to describe socioeconomic inequalities in temporal trends.

Results Preliminary results show that age-sex standardised annual prevalence increased from 32.9\% (95\% CI: $32.7 \%$ $33.1 \%$ ) with basic multimorbidity and $14.9 \%$ (95\% CI: $14.7 \%$ $15.0 \%)$ with complex multimorbidity in 2004 , to $51.0 \%(95 \%$ CI: $50.8 \%-51.3 \%)$ and $29.9 \%$ (95\% CI: $29.7 \%-30.1 \%)$ in 2019 , or by $55.3 \%$ and $101.0 \%$ respectively.

Basic multimorbidity incidence per 10,000 person-years showed little change from 644 (95\% CI: 631 - 658) in 2004 to $669(95 \%$ CI $648-690)$ in 2019 . There was an increase in the incidence of complex multimorbidity from $322(95 \%$ CI: $315-330$ ) to 418 (95\% CI: $407-430$ ).

The mean age of incident multimorbidity onset was 48.8 (95\% CI: 48.7 - 48.8) years for basic and 57.5 years $(95 \%$ CI: 57.5 - 57.6) for complex multimorbidity.

Conclusion The prevalence of both basic and complex multimorbidity has increased substantially over the last 15 years. Complex multimorbidity incidence and prevalence have increased more rapidly than for basic multimorbidity. This highlights the need for improved population-level prevention strategies to postpone and prevent the onset of long-term conditions. Our next step is to assess whether there are socioeconomic differences in these temporal trends.

\section{OP30 U.S. AND UK DIFFERENCES IN THE ASSOCIATION BETWEEN MULTIMORBIDITY TRAJECTORIES AND RETIREMENT IN OLDER WORKERS WITH HIGH AND LOW EFFORT-REWARD IMBALANCE}

M Mutambudzi*, E Demou, P Flowers. MRC/CSO Social and Public Health Sciences Unit, University of Glasgow, Glasgow, UK

\subsection{6/jech-2020-SSMabstracts.30}

Background Previous research has indicated that effort rewardimbalance (ERI) is independently associated with retirement and cross-sectional multimorbidity. In addition, aging populations and pension reform across western societies has led to older adults who are at increased risk of aging related disease onset and progression, participating in the labour force for longer. The objective of this study was to examine the association between multimorbid chronic health trajectories and retirement in older workers experiencing high and low effortreward imbalance.

Methods This study used longitudinal data from the Health and Retirement Study (2006-2016) and the English Longitudinal Study of Ageing (2004-2014) for adults aged 50-55 years. Group-based trajectory modelling was used to construct multimorbidity trajectories (0-6 of diabetes, hypertension, heart disease, stroke, lung disease and cancer) over a 10-year period separately for participants reporting low and high ERI at baseline. Logistic regression analysis fully adjusted for relevant variables examined the association between the multimorbidity trajectories and cross-sectional retirement at the final wave.

Results Mean ERI scores were higher in UK workers (low ERI $=0.75$, high ERI $=1.38$ ), compared to U.S. workers (low ERI $=0.67$, high ERI $=1.32$ ). Four trajectory classes were identified for U.S. workers with low ERI (no conditions, noincreasing, low-increasing, and high-stable). Compared to the no conditions trajectory, the high stable trajectory was associated with retirement $(\mathrm{HR}=4.50,95 \% \mathrm{CI}=2.08-9.62)$. Four trajectory classes (no-increasing, low-increasing, medium-stable, and high increasing) were identified for U.S. participants with high ERI. The medium-stable $(\mathrm{HR}=3.14,95 \% \mathrm{CI}=1.19-8.29)$ and high-increasing $(\mathrm{HR}=4.52,95 \% \mathrm{CI}=1.32-15.46)$ trajectories were associated with retirement. UK participants with high and low ERI were each classified into 3 trajectory classes respectively (no conditions, low-increasing, high-increasing), however no significant associations with retirement were observed.

Conclusion Our findings demonstrate country differences in mean ERI scores and trajectories of multimorbidities and their association with retirement. Trajectories with high intercepts indicating multimorbidity (i.e. $2+$ chronic conditions at baseline) were associated with retirement in U.S. older adults only, regardless of ERI. These results imply that baseline multimorbidity status may play a more important role than ERI on retirement in those approaching the retirement age, however further research is required. Socio-economic inequalities and social policies may provide partial explanations for these findings. Public health and workplace interventions may be warranted for workers with multimorbidities. 